Background Injuries in parasport are not well explored and may lead to detrimental effects in players with pre-existing disabilities. Previous parasport injury studies have focused on elite sledge hockey during winter Paralympics. Reported injury rates in sledge hockey are higher than other winter parasports.

Objective To examine concussion and injury rates, locations, types, mechanisms, and risk factors in sledge hockey.

Design Cross-sectional survey.

Setting Sledge hockey players across all levels of play.

Participants Sledge hockey players (ages $\geq 14$ years) recruited worldwide following the 2019-2020 season through email, social media and word of mouth communication. Ninety-two players initiated the survey and 77 (83.7\%) answered questions related to sledge hockey injuries.

Assessment of Risk Factors Risk factors considered participant demographics (e.g., age, sex, disability, level of play).

Main Outcome Measurements One-year injury rates (IR) and incidence rate ratios (IRR) were estimated based on univariate Poisson regression analyses. Injury proportions by type, location, and mechanism were described.

Results There were 47 injuries reported including 16 concussions in $9 / 77(11.7 \%)$ players and 31 non-concussion injuries in $20 / 77(26.0 \%)$ players. The overall IR was 13.2 injuries/ 1000 athlete-exposures (95\%CI;9.6-17.6). The game IR (28.4 injuries/1000 game-exposures, 95\%CI;18.6-41.7) was significantly higher than for practices (4.4 injuries/1000 practiceexposures, 95\%CI;2.2-7.9) $(\mathrm{IRR}=6.5$, 95\%CI;3.1-14.5). Significant injury: (1) locations were the head (34.0\%), wrist/ hand $(14.8 \%)$ and shoulder $(10.6 \%)$; (2) types were concussion (36.2\%) and bone fracture (8.5\%); and (3) mechanism was body checking $(42.1 \%$ of injuries caused by contact with another player). Age, sex, disability type, and level of play were not found to be injury risk factors.

Conclusions Concussions and upper extremity injuries were the most common sledge hockey injuries reported, with body checking being the most common mechanism. This research will inform the development of sledge hockey injury prevention strategies.

\section{WHO? WHAT? WHERE? WHY? DESCRIBING THE PATTERNS OF INJURY IN HIGH SCHOOL MALE COLLISION SPORTS}

\begin{abstract}
1,2Stephen West, ${ }^{1}$ Eric Gibson, ${ }^{1}$ Mark Patrick Pankow, 1,2,3,4Amanda Black, 1,2,3,4,5,6,7 Carolyn Emery. 'Sport Injury Prevention Research Centre, Faculty of Kinesiology, University of Calgary, Canada, Calgary, Canada; ${ }^{2} \mathrm{O}$ Brien Institute of Public Health, University of Calgary, Calgary, Canada; ${ }^{3}$ Alberta Children's Hospital Research Institute, University of Calgary, Canada, Calgary, Canada; ${ }^{4}$ Hotchkiss Brain Institute, University of Calgary, Canada, Calgary, Canada; ${ }^{5}$ McCaig Institute for Bone and Joint Health, University of Calgary, Canada, Calgary, Canada; ${ }^{6}$ Community Health Sciences, Cumming School of Medicine, University of Calgary, Canada, Calgary, Canada; 'Department of Paediatrics, Cumming School of Medicine, University of Calgary, Canada, Calgary, Canada
\end{abstract}

\subsection{6/bjsports-2021-IOC.413}

Background Collision sports (Canadian football, ice hockey, lacrosse, rugby) are popular among Canadian male youth, however it is recognised that collision sports are associated with a high risk of injury.

Objective To describe the patterns of collision sport-related injury in Canadian male high school athletes.

Design Secondary analysis of a cross-sectional survey.

Setting High schools (Alberta, Canada)
Participants 360 male students (of 2029 respondents), who play at least one of Football, Hockey, Lacrosse or Rugby.

Assessment of Risk Factors An anonymous online survey included questions regarding the mechanism, site, type, and nature of collision sport injuries.

Main Outcome Measurements Sport-related injury self-reported in the past year.

Results Of the 2029 survey respondents, 958 (47.2\%) were male of which $360(37.6 \%)$ reported playing at least one collision sport. Of all serious injuries reported by males, collision sports accounted for 33\% [hockey: 63(17\%), football: 41(11\%), lacrosse: 9(3\%), rugby $8(2 \%)]$. The head/face accounted for the largest proportion of injuries (hockey: $25.4 \%$, football: $24.4 \%$, lacrosse: $33.3 \%$, rugby $50.0 \%$ ). Concussion was the most common injury in rugby $(50.0 \%)$ and football (24.4\%) and fractures the most common in hockey $(27.0 \%)$ and lacrosse (44.4\%). Contact with another player was the most frequently reported mechanism of injury (rugby: 87.5\%, football: $77.1 \%$, lacrosse: $66.7 \%$, hockey: 57.4\%), with most injuries related to contact by a player who was bigger or the same size as the injured player (hockey/rugby:100\%, lacrosse: 83.3\%, football: 81.5\%).

Conclusions Sport-related injuries in male collision sports are common, with four sports accounting for 33\% of all reported injuries across male Canadian high school sports. Head/face injuries were the most common, with the majority of injuries occurring due to contact with another player. There is scope to consider primary prevention strategies such as contact training and rule changes to address the risk of injury in youth collision sport.

\section{ABSTRACT WITHDRAWN}

\section{HIGH LEVEL OF ORAL DISEASE AND SELF-REPORTED PERFORMANCE IMPACTS IN MALAYSIAN ELITE ATHLETES}

${ }^{1}$ Norul Husna Mohammad-Hassan, ${ }^{1}$ Nora Sakina Mohd Noor, ${ }^{1}$ Nor Shafina Mohamed Nazari, 'Zubaidah Zanul Abidin, ${ }^{1}$ Noor Hayati Azami, 2,3 lan Needleman. 'Department of Restorative Dentistry, Faculty of Dentistry, University of Malaya, Kuala Lumpur, Malaysia; ${ }^{2}$ Centre for Oral Health and Performance, University College London Eastman Dental Institute, London, UK; ${ }^{3}$ UK IOC Research Centre, University College London, London, UK

\subsection{6/bjsports-2021-IOC.414}

Background Oral health can affect performance, including athletes who always need to be in their best conditions during training and competitions. However, oral health awareness has never been a priority among Malaysian athletes.

Objective This study primarily aimed to assess the association between oral health status and self-reported impact on athletes' sports performance, using the Oral Health Screening Toolkit for Athletes developed by University College London, UK.

Design/Setting/Participants This is a cross-sectional study using convenience sampling. Data were collected among elite athletes registered under the National Sport Council (NSC), Malaysia. 